www.nature.com/ejhg

\title{
Is DFNA5 a susceptibility gene for age-related hearing impairment?
}

\author{
Lut Van Laer ${ }^{1}$, Anita L DeStefano ${ }^{2,3}$, Richard H Myers ${ }^{3}$, Kris Flothmann ${ }^{1}$, Sofie Thys ${ }^{1}$, \\ Erik Fransen ${ }^{1}$, George A Gates ${ }^{4}$, Guy Van Camp*,1 and Clinton T Baldwin ${ }^{5}$
}

${ }^{1}$ Department of Medical Genetics, University of Antwerp, Antwerp, Belgium; ${ }^{2}$ Department of Biostatistics, Boston University School of Public Health, Boston, Massachusetts, USA; ${ }^{3}$ Department of Neurology, Boston University, Boston, Massachusetts, USA; ${ }^{4}$ Virginia Merrill Bloedel Hearing Research Center, University of Washington, Seattle, Washington, USA; ${ }^{5}$ Center for Human Genetics, Boston University School of Medicine, Boston, Massachusetts, USA

\begin{abstract}
A mutation in DFNA5 leads to a type of hearing loss that closely resembles the frequently observed agerelated hearing impairment (ARHI). The hearing loss is sensorineural, progressive and starts at the high frequencies. As DFNA5 was considered an excellent candidate ARHI susceptibility gene, we performed linkage analysis to a quantitive measure of high frequency hearing loss. However, no significant linkage between ARHI and microsatellite markers from the DFNA5 region could be detected. Subsequently, the DFNA5 coding region was analysed for single nucleotide polymorphisms (SNPs). Two SNPs leading to amino-acid substitutions (P142H and V207M) were selected for further analysis. Using these SNPs, an association study based on a collection of random individuals, and a case-control association study were performed. No significant differences in genotypes between good hearing and hearing impaired individuals could be detected in either study design. We conclude that there exists no strong association between DFNA5 and ARHI.
\end{abstract}

European Journal of Human Genetics (2002) 10, 883 -886. doi:10.1038/sj.ejhg.5200878

Keywords: DFNA5; SNP; age-related hearing impairment; association study

\section{Introduction}

Hearing impairment is the most frequent sensory handicap. A significant hearing loss of more than 25 decibels is found in $15-20 \%$ of the adult population. This prevalence is increasing with age, reaching approximately $50 \%$ by the age of $80 .{ }^{1}$ Age-related hearing impairment (ARHI) is multifactorial, displaying both environmental and genetic components. Well-documented environmental factors include ototoxic drugs, infections and acoustic trauma. The contribution of tobacco smoking, exposure to solvents, and long-term usage of certain drugs remains controversial. The role of genetic factors in the development of ARHI was only recently established. A Swedish male twin population was studied, and a heritability estimate of 0.47 was calculated for the age group above 65 years, indicating that

*Correspondence: G Van Camp; Department of Medical Genetics, University of Antwerp, Universiteitsplein 1, B-2610 Antwerp, Belgium. Tel: 323-820.25.85; Fax: 323-820.25.66; E-mail: gvcamp@uia.ua.ac.be Received 26 March 2002; revised 17 July 2002; accepted 18 July 2002 approximately half of the variance is caused by genetic differences. ${ }^{2}$ Another study compared the auditory status in genetically unrelated (spouse pairs) and genetically related (sibling pairs, parent-child pairs) subjects. The heritability estimates of this study suggested that $35-55 \%$ of the variance of sensory ARHI is attributable to the effects of genes. $^{3}$

Up to now, nothing is known about the nature of the genes that contribute to ARHI. As the most frequent type of ARHI is progressive, sensorineural and most pronounced in the high frequencies, genes causing monogenic hearing impairment with phenotypic similarities to ARHI (although with a much younger age-at-onset) are excellent candidate ARHI susceptibility genes. A complex mutation in DFNA5 is responsible for the hearing impairment in an extended Dutch family. The hearing loss is sensorineural and progressive, and starts in the high frequencies at an age between 5 and 15 years. ${ }^{4}$ Although the mutation segregating in the Dutch family remains the only DFNA5 mutation described hitherto, we believe that DFNA5 might constitute one of 
the genetic factors involved in ARHI. In this study we have investigated the hypothesis that DFNA5 is one of the susceptibility genes for ARHI by quantitative trait linkage analysis in extended pedigrees as well as by two different association studies using two DFNA5 single nucleotide polymorphisms (SNPs).

\section{Materials and methods Random individuals}

A detailed description of the audiometric methodology and the selection criteria will be published elsewhere. Briefly, pure-tone audiometry with air conduction at 250, $500,1000,2000,4000,8000 \mathrm{~Hz}$ and bone conduction at 250, 500, 1000, 2000, $4000 \mathrm{~Hz}$ was performed on 133 random Caucasian individuals between 40 and 80 years. On the basis of the ISO 7029 standard (ISO 7029, International Organisation for Standardisation, Geneva 1984, 'Acoustics-threshold of hearing by air conduction as a function of age and sex for otologically normal persons'), the frequency specific thresholds were converted to sexand age-independent hearing standard deviations (HSD). The HSD at a particular frequency is defined as the number of standard deviations the hearing threshold differs from the median value. In persons belonging to the better hearing half of the population, this value is negative. For each subject the better ear was selected by comparing the sum of the HSDs at 2000, 4000 and $8000 \mathrm{~Hz}$ for each ear, and all subsequent calculations were performed on the better ear. Conductive losses were expressed as the mean air-bone threshold differences at 500, 1000 and $2000 \mathrm{~Hz}$. When conductive losses exceeded $10 \mathrm{~dB}$, the subject was excluded from the study. Noise dips were calculated as the difference between air thresholds at 4000 and $8000 \mathrm{~Hz}$, and were only allowed if they were smaller than $20 \mathrm{~dB}$. For each remaining subject (116 in total), pure-tone HSD averages at 2000, 4000, and $8000 \mathrm{~Hz}\left(\mathrm{PTA}_{2,4,8 \mathrm{KHz}}\right)$ were calculated.

\section{Pedigrees and case-controls}

Extended pedigrees for linkage analysis and sensory hearing loss cases and normal hearing controls were selected from the National Heart Lung and Blood Institute's Framingham Heart Study. The Framingham Heart Study is a population based sample with initial recruitment of 5209 men and women from Framingham, Massachusetts, USA beginning in $1948^{5}$ (http://rover2.nhlbi.nih.gov/about/framingham/ index.html). Starting in 1971, 5124 offspring and spouses of offspring of original participants were recruited. ${ }^{6}$ In 1973-1975 hearing examinations were conducted on 2263 members of the original cohort (exam cycle 15) and in 1995-1999 identical exams were conducted on 2217 members of the offspring (exam cycle 6). All subjects gave informed consent and the study protocol was approved by the Institutional Review Board at Boston Medical Center (Boston, MA) and the University of Washington.
Standard pure-tone audiograms were obtained on all subjects using environments and methods meeting ANSI (American National Standards Institute) standards. The behavioural audiometric thresholds were averaged for the low, mid, and high frequencies. The high frequency pure tone average (PTAhigh), which is the trait of interest in this study, consisted of the thresholds obtained at 4000, 6000 and $8000 \mathrm{~Hz}$. Based on threshold level and shape of the audiometric curve, an audiometric classification was done using an algorithm to smooth and categorize each ear's hearing into one of 10 'phenotypes'. Type 0 was normal hearing, types 1-3 were 'sensory', types 4-5 were 'strial', and the remainder were 'other'. The subjects were grouped by type of ARHI; within each type, increasing number indicates poorer hearing. ${ }^{3}$

Of the Framingham Heart Study subjects with hearing data there were 1789 individuals that were members of 328 pedigrees used for linkage analysis in the DFNA5 region. Additionally, 93 unrelated individuals with hearing loss (type 1, 2 or 3 in both ears) and 83 unrelated individuals with normal hearing (type 0 in both ears) were selected for association studies. The individuals with normal hearing were restricted to those aged 50 or older at the time of audiometric exam. Mean age of normal hearing controls $(58.0 \pm 4.40)$ was older than that of the cases with sensory hearing loss $(55.0 \pm 4.38)(P<0.0001)$.

\section{Linkage analysis}

Genotyping of microsatellite markers was performed by the Mammalian Genotyping Service laboratory at the Marshfield Clinic (Marshfield, WI). A $10 \mathrm{~cm}$ density genome scan was performed (marker set $8 \mathrm{~A}$, average heterozygosity 0.77). Only markers in the region of DFNA5 [D72210 (33 cM), D7S1808 (42 cM) and D7S817 (50 cM)] were examined in this study. Details regarding markers and primers are available from Research Genetics (http://www.marshmed.org/genetics/default.htm). Mendelian inconsistencies were detected using the GENTEST program (http:// www.sfbr.org/sfbr/public/software/software.html) and genotype information was set to 'missing' for all members of nuclear families for a marker in which an inconsistency was detected. Variance component linkage analysis was performed using the program SOLAR. ${ }^{7}$ The quantitative measure of ARHI used for linkage analysis was the standardised residual of PTAhigh in the best ear adjusted for cohort, sex, age, age to the second, and age to the third power.

\section{SNP identification and analysis}

To identify coding DFNA5 SNPs, exons 2 to 9, and the translated part of exon 10 were sequenced in 10 random individuals using the primers listed in Table 1. PCR products generated using standard methods and an annealing temperature of $55^{\circ} \mathrm{C}$, were purified with the QIAquick PCR Purification Kit (Qiagen). Sequencing reactions were 
Table 1 Sequencing primers

\begin{tabular}{llc}
\hline Name & Sequence $\left(5^{\prime} \rightarrow 3^{\prime}\right)$ & Length of product $(\mathrm{bp})$ \\
\hline Exon-2-F & Gactaattatagaatatacccaagg & 278 \\
Exon-2-R & Ctttacttttcagataatctggag & 254 \\
Exon-3-F & Ccaaaatgccaaacaagtctcc & \\
Exon-3-R & Catcggcttcctctgctcg & 246 \\
Exon-4-F & Gctcagccatgaacgcagg & \\
Exon-4-R & Gcactaagccactcacatcc & 182 \\
Exon-5-F & Cctcagcacccataggagg & 242 \\
Exon-5-R & Gcacaggtgggagtgtgcg & 187 \\
Exon-6-F & Caactgcccactactcctgc & \\
Exon-6-R & Gactcaactttacctaacttgtcc & 266 \\
Exon-7-F & Gccttgagatgtctcagggc & \\
Exon-7-R & Cagtcatgagacttgggtgtgc & 208 \\
Exon-8-F & Ccatttctttcatttcttttctcc & \\
Exon-8-R & Gtctccagctgtgtcatgacc & \\
Exon-9-F & Cggctgctggatgtctacc & \\
Exon-9-R & Gctgtcttccatggacttg & \\
Exon-10-F & Caactttaacgtgcatatgacc & \\
Exon-10-R & Cctacaagtgcattactatcc & \\
\hline
\end{tabular}

performed using the ABI Prism Big Dye Terminator Cycle Sequencing Kit on an ABI 377 automated sequencer. SNP genotyping was carried out by PSDM (PCR-mediated sitedirected mutagenesis). This was performed using standard PCR conditions with 5'-gcacctgctggagcccag-3' (modified primer) and 5'-ctcagctgcctgctgtgc-3' for the P142H SNP, and 5'-aatgtcaccaaggactcccac-3' (modified primer) and Exon-5-F (Table 1) for the V207M SNP. The respective annealing temperatures were $59^{\circ} \mathrm{C}$ and $57^{\circ} \mathrm{C}$. PCR products were digested with MvaI (Fermentas) or Eco72I (Fermentas) respectively, and fragments were analysed by electrophoresis on a $12 \%$ polyacrylamide gel.

\section{Statistical analysis}

In the association study on random individuals, $\mathrm{PTA}_{2,4,8 \mathrm{kHz}}$ and SNP genotypes were analysed using one-way analysis of variance (ANOVA) using The Sigma Stat 2.0 software (Jandel Scientific Corporation) . In the case-control association study on the Framingham samples, differences between groups were tested with $\chi^{2}$ statistics using SAS (v8.02).

\section{Results}

Variance component linkage analysis was performed resulting in a maximum two-point LOD score of 0.69 at D7S2210. The LOD score was 0.00 at the two other markers examined. The maximum multipoint LOD score across this region was 0.136 at $30 \mathrm{~cm}$.

Sequencing of the DFNA5 coding region in 10 random individuals resulted in the detection of 6 SNPs (Table 2). Two of these SNPs, $481 \mathrm{C} \rightarrow \mathrm{A}$ and $676 \mathrm{G} \rightarrow \mathrm{A}$, lead to amino acid alterations (P142H and V207M) and were selected for further analysis. A PSDM was designed (Figure 1 ), and the allele frequency was determined in 50 Belgian controls. An allele frequency of $80 \%$ for the $\mathrm{C}$ allele for
Table 2 DFNAS polymorphisms

\begin{tabular}{|c|c|c|}
\hline base $^{a}$ & Exon & Amino acid \\
\hline $481 C \rightarrow A$ & Exon 4 & P142H \\
\hline $504 \mathrm{~A} \rightarrow \mathrm{G}$ & Exon 4 & E149E \\
\hline $546 \mathrm{G} \rightarrow \mathrm{A}$ & Exon 4 & T163T \\
\hline $676 \mathrm{G} \rightarrow \mathrm{A}$ & Exon 5 & V207M \\
\hline $921 \mathrm{G} \rightarrow \mathrm{A}$ & Exon 7 & A288A \\
\hline $1257 \mathrm{G} \rightarrow \mathrm{A}$ & Exon 9 & A400A \\
\hline
\end{tabular}

A $\begin{array}{lllllll}M & 1 & 2 & 3 & 4 & 5 & 6\end{array}$

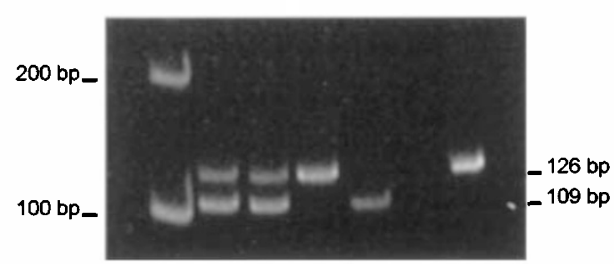

B $\begin{array}{lllllll}M & 1 & 2 & 3 & 4 & 5 & 6\end{array}$

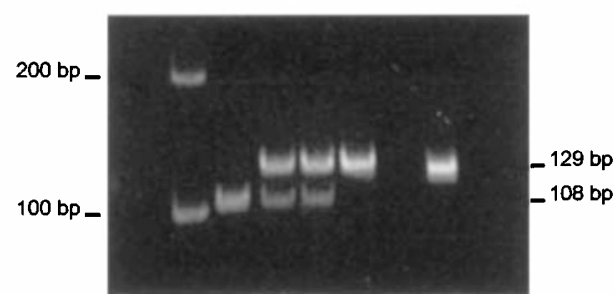

Figure 1 Typical P142H and V207M analysis in ethidium bromide stained $12 \%$ polyacrylamide gels. (A) $\mathrm{P} 142 \mathrm{H}$ analysis using PSDM with Mval on two heterozygotes (lane 1 and 2), one homozygote for the A allele (lane 3) and one homozygote for the $C$ allele (lane 4). Lane 5 contains a PCR control reaction without template and lane 6 contains undigested PCR product. (B) V207M analysis using PSDM with Eco72l on one homozygote for the $\mathrm{G}$ allele (lane1), two in heterozygotes (lane 2 and 3 ) and one homozygote for the A allele (lane 4). Lane 5 contains a PCR control reaction without template and lane 6 contains undigested PCR product.

$\mathrm{P} 142 \mathrm{H}$ and of $93 \%$ for the $\mathrm{G}$ allele for V207M was found, indicating that both polymorphisms were informative and could be used for the subsequent ARHI association studies.

A pilot experiment on 116 random Belgian and Dutch subjects was initiated, but no association could be detected between either SNP and ARHI (Table 3). Subsequently, 93 ARHI cases and 83 controls, selected from the Framingham cohort, were genotyped for $\mathrm{P} 142 \mathrm{H}$. The observed frequency for the A allele was 18\% in the control group, and 13\% in the group with ARHI $(P=0.179)$. No allelic or genotypic association was detected (Table 4 ). 
Table 3 Results of the association study on randomly collected subjects

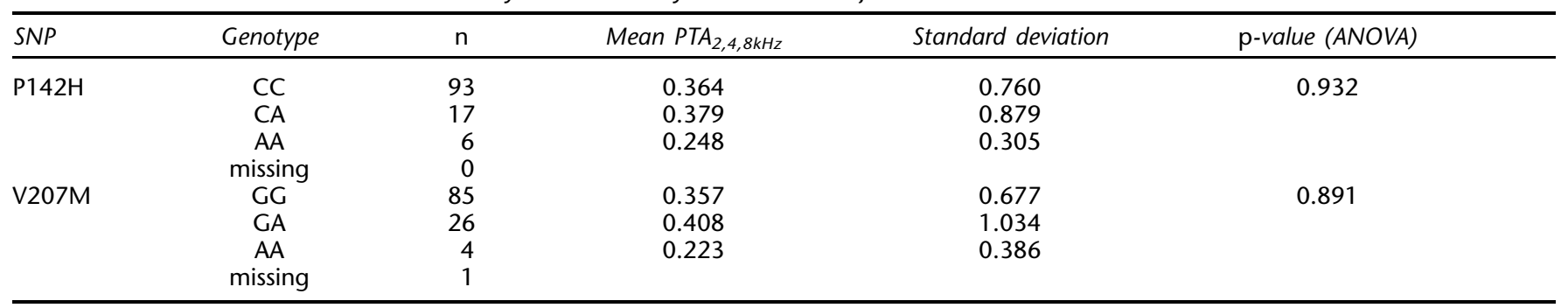

Table 4 Results of the association study in subjects with and without sensory hearing loss from the Framingham Heart Study

\begin{tabular}{|c|c|c|c|c|c|}
\hline \multirow[b]{2}{*}{ Subjects } & \multicolumn{4}{|c|}{ P142H Genotype } & \multirow{2}{*}{$\begin{array}{c}P \text {-value } \\
\text { (Chi-square) }\end{array}$} \\
\hline & $n$ & CC & $C A$ & $A A$ & \\
\hline Sensory cases & 93 & $\begin{array}{c}72 \\
(77.4 \%)\end{array}$ & $\begin{array}{c}18 \\
(19.4 \%)\end{array}$ & $\begin{array}{c}3 \\
(3.2 \%)\end{array}$ & 0.422 \\
\hline Controls & 83 & $\begin{array}{c}57 \\
(68.7 \%)\end{array}$ & $\begin{array}{c}22 \\
(26.5 \%)\end{array}$ & $\begin{array}{c}4 \\
(4.8 \%)\end{array}$ & \\
\hline
\end{tabular}

\section{Discussion}

As a mutation in DFNA5 leads to a type of hearing impairment that closely resembles the most frequently observed ARHI phenotype, namely a sensorineural, progressive hearing impairment that is most pronounced in the high frequencies, DFNA5 was considered an excellent candidate ARHI susceptibility gene. However, no significant linkage of ARHI and DFNA5 could be detected using quantitative trait variance component linkage analysis. Linkage analysis in sample sizes as used in this study can only detect susceptibility genes with a moderate or strong effect. Case-control association studies are more powerful to detect weak effects. ${ }^{8}$ Therefore, despite of negative linkage data, we considered an association study to be worthwhile.

For the association studies, two DFNA5 SNPs leading to amino acid substitutions were selected. These SNPs have the advantage that the observed association might be due to a direct causative effect, rather than linkage disequilibrium. However, both in a study on randomly collected Caucasian subjects and in a case-control study on subjects selected from the Framingham cohort, no significant association between DFNA5 and ARHI could be detected. Monogenic hearing impairment is extremely heterogeneous (Van Camp G, Smith RJH, Hereditary Hearing Loss Homepage, URL:http://dnalab-www.uia.ac.be/dnalab/hhh/), with more than 70 known loci and many more localisations and identifications expected. If the situation for ARHI is similar, it is possible that many different genes contribute to ARHI, each with a weak effect. In this case, associations between candidate genes and ARHI might be difficult to detect and ARHI might turn out to be too complex to study with moderate sample sizes. On the other hand, it might be that by using much larger sample sizes, associations between candidate genes and ARHI could be detected.

In conclusion, this study could not detect a significant association between DFNA5 and ARHI using moderate sample sizes, suggesting the absence of a major effect. Future collaborative studies on a large number of ARHI samples should be performed in order to detect possible weak effects.

\section{Acknowledgements}

GVC and LVL hold research positions with the Flemish FWO (Fonds voor Wetenschappelijk Onderzoek). This work was supported by the National Institutes of Health grant DC01525 (to GA Gates and CT Baldwin). We would also like to thank the NIH-NHLBI supported Framingham Heart Study for their cooperation.

\section{References}

1 Morton NE: Genetic epidemiology of hearing impairment. Ann NY Acad Sci 1991; 630: 16-31.

2 Karlsson KK, Harris JR, Svartengren M: Description and primary results from an audiometric study of male twins. Ear Hear 1997; 18: $114-120$.

3 Gates GA, Couropmitree NN, Myers RH: Genetic associations in age-related hearing thresholds. Arch Otolaryngol Head Neck Surg 1999; 125: 654-659.

4 Van Laer L, Huizing EH, Verstreken $\mathrm{M}$ et al: Nonsyndromic hearing impairment is associated with a mutation in DFNA5. Nature Genet 1998; 20: 194-197.

5 Dawber TR, Meadors GF, Moore FEJ: Epidemiological approaches to heart disease: the Framingham Study. Am J Public Health 1951; 41: $279-286$.

6 Kannel WB, Feinbleib M, McNamara PM, Garrison RJ, Castelli WP: An investigation of coronary heart disease in families: the Framingham Offspring Study. Am J Epidemiol 1979; 110: 281-290.

7 Almasy L, Blangero J: Multipoint quantitative trait linkage analysis in general pedigrees. . Am J Hum Genet 1998; 62: 1198-1211.

8 Risch N, Merikangas K: The future of genetic studies of complex human diseases. Science 1996; 273: 1516-1517. 